\title{
Perinatal mortality associated with use of uterotonics outside of Comprehensive Emergency Obstetric and Neonatal Care: a cross-sectional study
}

\author{
Louise T. Day ${ }^{1,2^{*}}$, Daniel Hruschka ${ }^{3}$, Felicity Mussell ${ }^{4}$, Eva Jeffers ${ }^{3}$, Stacy L. Saha ${ }^{1}$ and Shafiul Alam ${ }^{1}$
}

\begin{abstract}
Background: Prior studies have shown that using uterotonics to augment or induce labor before arrival at comprehensive Emergency Obstetric and Neonatal Care (CEmONC) settings (henceforth, "outside uterotonics") may contribute to perinatal mortality in low- and middle-income countries. We estimate its effect on perinatal mortality in rural Bangladesh.
\end{abstract}

Methods: Using hospital records (23986 singleton term births, Jan 1, 2009-Dec 31, 2015) from rural Bangladesh, we use a logistic regression model to estimate the increased risk of perinatal death from uterotonics administered outside a CEmONC facility.

Results: Among term births ( $\geq 37$ weeks gestation), the risk of perinatal death adjusted for key confounders is significantly increased among women reporting uterotonic use outside of $C E m O N C(O R=3 \cdot 0,95 \% \mathrm{Cl}=2 \cdot 4,3 \cdot 7)$. This increased risk is particularly high for fresh stillbirths $(\mathrm{OR}=4 \cdot 0,95 \% \mathrm{Cl}=3 \cdot 0,5 \cdot 3)$ and intrapartum-related causes of early neonatal deaths (birth asphyxia) $(\mathrm{OR}=3 \cdot 1,95 \% \mathrm{Cl}=2 \cdot 2,4 \cdot 5)$.

Conclusions: In this sample, outside uterotonic use was associated with substantially increased risk of fresh stillbirths, deaths due to birth asphyxia, and all perinatal deaths. In settings of high uterotonic use outside of controlled settings, substantial improvement in both stillbirth and early neonatal mortality may be made by reducing such use.

Keywords: Perinatal mortality, Uterotonics, Bangladesh, Stillbirth, Neonatal death, Child survival, Under-five mortality

\section{Plain English summary}

Uterotonics can be used safely to treat disorders of labor in a delivery hospital where the mother can be monitored and complications can be treated. But in rural Bangladesh, uterotonics are also widely available and are often used outside of such controlled settings. We found that more babies were born dead or died during their first week of life if their mothers told us that they had received uterotonics before arriving at a safe delivery hospital. If fewer mothers received uterotonics to speed

\footnotetext{
* Correspondence: louisetday@gmail.com

${ }^{1}$ LAMB MIS-Research Department, Parbatipur, Dinajpur 5250, Bangladesh

2LAMB Hospital Pediatric Department, Parbatipur, Dinajpur 5250, Bangladesh
}

Full list of author information is available at the end of the article labour outside of controlled settings, many babies' deaths can potentially be prevented.

\section{Background}

Globally each year, an estimated $2 \cdot 6$ million newborns die [1], and another 2.6 million babies are stillborn, half of whom die during labour and birth [2,3]. The vast majority ( $>98 \%$ ) of these deaths occur in low- and middleincome countries $[3,4]$. With other causes of under-five mortality declining, neonatal deaths now account for $44 \%$ of all under-five deaths making them a crucial focus for improving child survival [5-7]. Renewed calls to action to end these largely preventable child deaths include addressing gaps in knowledge about stillbirth 
risk factors and stillbirth prevention as well as preventing deaths due to birth asphyxia $[3,7,8]$.

In recent years, practitioners in low- and middleincome countries have raised concerns that the use of uterotonics, an otherwise life-saving treatment, outside of controlled settings is contributing to these perinatal deaths [9]. Induction and active management of labor with uterotonics is a well-established and safe medical procedure as long as it is conducted in tightly controlled situations where one can identify correct indications of risk, precisely administer doses, frequently monitor fetal heart rate $[9,10]$ and perform emergency Cesarean section when necessary, namely in a CEmONC facility that follows appropriate procedures. In addition to facilitating active management of labor and reducing the complications of prolonged labour, uterotonics are also an important tool in preventing and treating post-partum haemorrhage, a leading cause of maternal death in lowand middle-income countries. Uterotonics can save lives and manage risk in clinical settings, but they can also threaten the lives of mothers and babies when used to augment or induce labor in settings without the appropriate resources and adherence to protocols. Such situations include home birth settings and basic EmONC facilities without full CEmONC support (henceforth, "outside uterotonic use"). These outside utertonics are frequently administered either as a bolus intravenous infusion without strict control of the infusion rate or as intramuscular injections. Notable consequences of "outside uterotonic use" include fetal hypoxia, neonatal encephalopathy or death, uterine rupture, and maternal death [9]. However, little systematic evidence has estimated the relative contribution of outside uterotonic use to perinatal outcomes [11], and currently none exists for stillbirth. The perceived benefit to the family of outside uterotonics use in Bangladesh includes speeding up labor, shortening labor duration, facilitating delivery, avoiding hospital delivery and reducing maternal suffering [12]. Given estimates of home-based uterotonic use ranging from 10 to $68 \%$ in low- and middle-income settings [9-13], there have been a number of recent calls for rigorous studies to quantify the magnitude and effect of inappropriate augmentation on perinatal and maternal outcomes [9-12, 14, 15].

This cross-sectional study uses seven years of hospital records in a low-income setting in rural, northwestern Bangladesh to estimate the increased risk of several perinatal birth outcomes, including stillbirths, from the reported use of injectable uterotonics to induce or augment labor outside of CEmONC facilities. We test the hypothesis that outside uterotonic use will have negative effects on several, but not all birth outcomes. Specifically, it should increase the risk of intrapartum (i.e. "fresh") stillbirth but not antepartum stillbirths (i.e. "macerated"). It should also increase the risk of early neonatal death (early NND) especially death caused by birth asphyxia. We assess this hypothesis against the null hypothesis of no increased risk among those mothers administered outside uterotonics.

\section{Methods \\ Setting}

LAMB Hospital is a CEmONC facility serving the rural poor in Northwest Bangladesh. On average 3500 babies are delivered at the hospital each year. During the study period, $5.7 \%$ of all mothers experienced prolonged labor, $3.1 \%$ breech deliveries, and $1.6 \%$ eclampsia. Approximately one fifth $(21.7 \%)$ were treated with Cesarians. The median birthweight was $2.8 \mathrm{~kg}$ (1st quartile $=2.5 \mathrm{~kg}$, 3rd quartile $=3.1 \mathrm{~kg}$ ). The hospital has developed and trained medical providers in the use of a simple interactive checklist medical record system "circle sheets" for documenting key maternal and neonatal clinical information before, during and after delivery including history, examination, and diagnoses. In addition to facilitating real-time medical decision-making, the circle sheets are then entered into a customised database, checked and cleaned for use in standard reporting and clinical audits. For variables considered here, circle sheets with fixed responses require the practitioner to specify at least one response (e.g. Yes, No, or Unknown), and missing data are relatively rare. All data analyses here are generated by an ongoing hospital data system that was originally developed in 2006 along with a number of quality monitoring, feedback and training practices aimed at improving completeness and accuracy of data by midwives and clinicians. These include crosscomparing data entry, regular checks on completeness of data, on-ward observations of form completion, and regular retraining personnel It also involves frequent data reports to hospital staff and communication with staff about any changes in the understanding or completion of the interactive checklist records.

\section{Sample}

There were 24935 babies delivered at LAMB Hospital between January 1st, 2009 and December 31st, 2015. For these analyses, we exclude twins and triplets $(2.9 \%$ of births, $n=724)$ as well as babies with birthweights $<1000 \mathrm{~g}(1 \cdot 1 \%$ of births, $n=264)$ and without information on gestational age $(n=19)$ (Total excluded $n=949)$. Pre-term births have a higher risk of adverse outcomes from prematurity related causes. Thus, we analyse preterm births ( $\leq 36$ week) separately $(6 \cdot 3 \%$ of total births, $n=1560$ ) and present these analyses in the additional information (see Additional file 1: Table S1). Pre-term births were classified as $\leq 36$ week gestation using a validated method based on 8 external characteristics [16]. 
This leaves a final total sample of 22426 term singleton births $\geq 37$ weeks and $\geq 1000$ g. Of these 22426 births, $2177(9.7 \%)$ were admitted infants whose guardians discharged them without doctor's approval, and thus were not available for follow-up in the case of complications. Analyses excluding these early discharged newborns $(n=20249)$ are not substantially different from those with the full sample (see Additional file 1: Table $\mathrm{S} 2$ for results on this subsample). We report results for the full sample of 22426 singleton term births in the main body. The flow diagram in Fig. 1 summarizes the excluded babies and the analysis pathways.

\section{Variables}

Birth outcomes We examined five birth outcomes prior to discharge: (1) fresh stillbirth, (2) macerated stillbirth, (3) early neonatal death (before discharge), (4) early neonatal death due to intrapartum-related complications (birth asphyxia), as well as a (5) composite outcome of perinatal death (stillbirth and early neonatal death).

Stillbirth type (fresh or macerated) was determined by the midwife or doctor who delivered the baby and recorded in the mother's chart and perinatal death form at the time of birth. Live born babies were either admitted to routine postnatal care by midwives or admitted under pediatric doctors according to strict clinical criteria including: sepsis risk, less than $2 \mathrm{~kg}$ birth weight, specific maternal risk factors, growth restriction, significant jaundice, congenital abnormalities, respiratory distress, weight loss $>10 \%$, resuscitation needed after birth, and feeding difficulties among other indications. Infants that died immediately after birth were assessed and the cause of death documented. The $67 \%$ of infants admitted to the hospital for routine postnatal care by midwives were determined free of these problems after a postnatal check. Each of the $33 \%$ of infants admitted to the hospital under pediatric doctors was classified as either alive at discharge or early neonatal death (death within 7 days of delivery prior to discharge). Admitted babies length of stay was median $2 \cdot 0$ days and mean 2.5 days. For all early neonatal deaths, a senior obstetrician and senior pediatrician reviewed the mother and baby's case notes as part of a monthly perinatal death audit. Causes of death were coded by these senior clinicians with the "Perinatal Problem Identification Programme (PPIP)", originally designed and developed in South Africa as a facility audit tool for perinatal deaths [17]. The PPIP has 5 subcodes for hypoxia (hypoxia, hypoxic ischemic encephalopathy, meconium aspiration, persistent fetal circulation and hypoxia other) which we attribute to intrapartum-related complications (birth asphyxia). All data was extracted from the hospital database, Flow Information System Hospital or FISH [18].

Reported outside uterotonic use At admission to the CEmONC unit, all mothers were routinely asked by the admitting midwife or doctor whether intramuscular injections or drips had already been administered to increase labor pains before arrival at this hospital. Mothers come to this CEmONC either from home or local basic EmONC facilities and only rarely are they given referral papers with details of treatment administered. Injectable uterotonics such as oxytocin are widely available in Bangladesh and use outside the CEmONC is

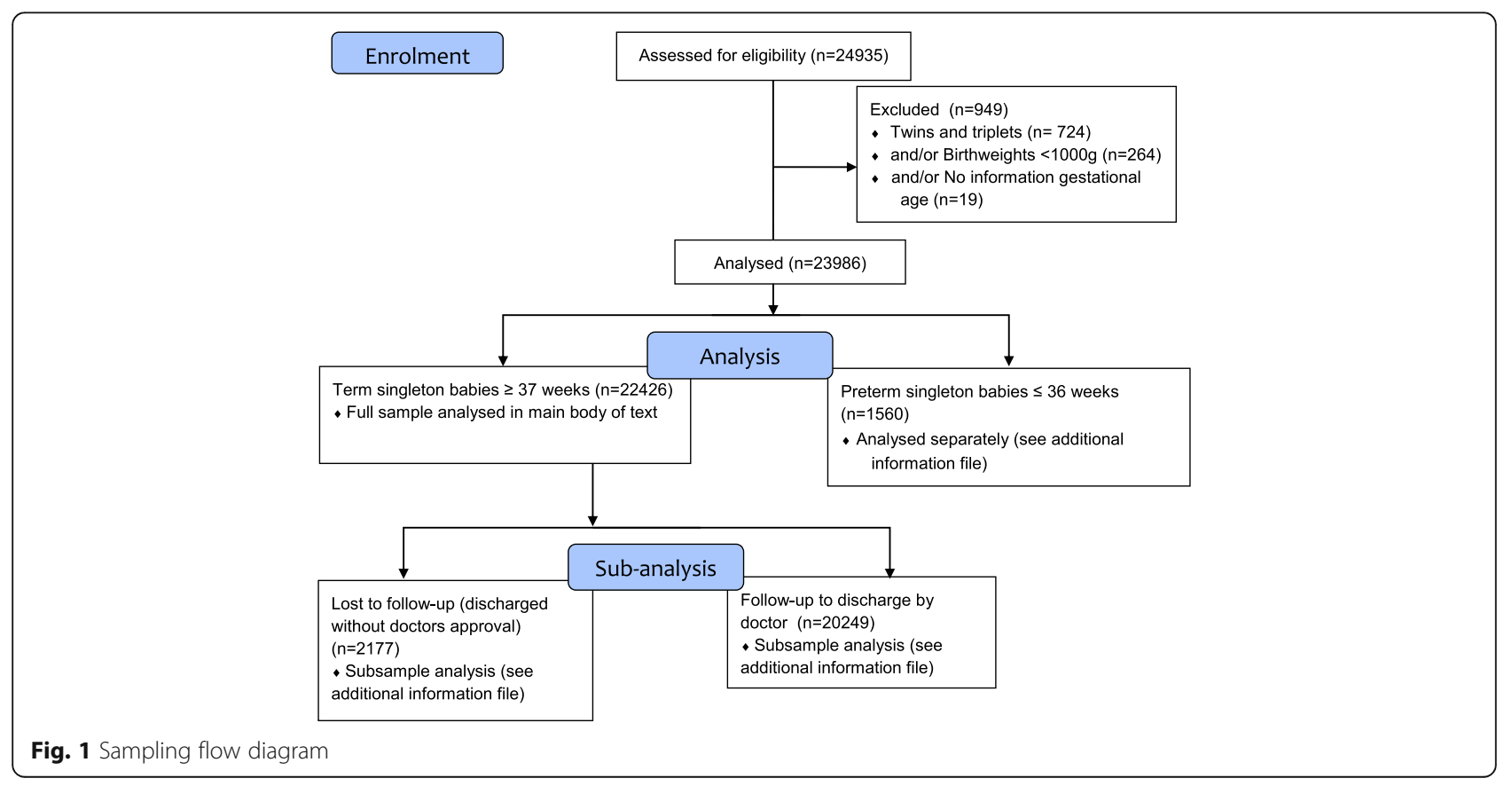


usually by an intramuscular injection or in an intravenous infusion or drip without any monitoring [12]. If mothers stated they had received injections or drip prior to coming to the hospital, either affirmatively or in terms of the number of treatments, this was documented on their clinical records and subsequently entered into the database coded as 1 for "outside uterotonic use". Otherwise they were coded as 0 . It was not documented whether the outside injectable uterotonic use had been in the home or at a Basic EmONC facility. Nor was the provider of the outside uterotonic documented eg the Village Doctor - a type of unqualified allopathic provider who usually operates in the home setting [12] or a medically trained provider in the basic EmONC setting.

Covariates We considered five potential covariates-prolonged labor, pre-eclampsia, eclampsia, induction or augmentation by uterotonics in the CEmONC hospital setting (LAMB Hospital), and Cesarian section.

Prolonged labor is a potential confounder of any observed relationship between outside uterotonic use and birth outcomes as prolonged labor can both: (1) lead caregivers to seek uterotonics in the home or in non-comprehensive EmONC facilities and (2) precipitate birth complications including intrapartum-related neonatal complications (birth asphyxia). For approximately $90 \%$ of births, health workers documented information on when the mother's first stage of labor had begun. This information was from mother's self-report if she presented at the hospital already in the first stage of labor or from the partograph if her first stage started after admission. The duration of labor was calculated by subtracting the beginning of first stage labor from the time of delivery. A categorical prolonged labor variable was created with labor $<12 \mathrm{~h}=1$, labor $12-24 \mathrm{~h}=2$, labor $>24 \mathrm{~h}=3$, and labor time is missing $=4$.

Eclampsia and pre-eclampsia are additional complications that may be confounding variables between outside uterotonic use and deaths due to birth asphyxia. Thus, we control for the effect of two levels of pre-eclampsia (moderate and severe) and eclampsia diagnosed by a clinical checklist. In this context, both CEmONC facility uterotonics to induce or augment and Cesarian section may arise from prolonged labor which could both precipitate outside uterotonic use prior to arrival at the EmONC facility and increase the risk of perinatal mortality. Thus we include these two procedures as proxies for potential confounders. Uterotonic induction or augmentation in LAMB Hospital is initiated by an Obstetric doctor for inadequate progress on the partograph. Uterotonic induction or augmentation at LAMB Hospital is documented in the maternal notes and entered on the database as a categorical variable: Yes, No or Unknown. Intermittent fetal heart monitoring is performed every 15 min for labors being induced or augmented with uterotonics. Cesarian section was recorded in the maternal birth chart and entered on the database as $1=$ Cesarian section and $0=$ no Cesarian section.

\section{Analysis}

We report raw rates of five outcomes-fresh stillbirth, macerated stillbirth, early neonatal death (before discharge), deaths due to birth asphyxia, and a fifth composite outcome of total perinatal deaths-for both the entire sample and the sub-sample reporting uterotonic use outside of CEmONC. We use a logistic regression to estimate the effect of outside uterotonic use on these outcomes, reporting both a crude, unadjusted odds ratios and odds ratios adjusted for potential covariatesprolonged labor, pre-eclampsia, eclampsia, uterotonic administration at the hospital, and Cesarian section. There were no cases of macerated stillbirth when a mother experienced eclampsia, so that variable was not included in the final macerated stillbirth model. Analyses were conducted in SPSS 22.0.

We estimated the population attributable risk percent from outside uterotonics using formula 4 in Rockhill and Newman applied to the adjusted relative risk [19]. Specifically, we calculate the population attributable risk percent $p d\left(\frac{R R-1}{R R}\right)$ where $\mathrm{pd}$ is the proportion of cases exposed to the risk factor and RR is the risk ratio associated with that exposure. The prevalence of all outcomes is less than $5 \%$ in the population. Thus, we use adjusted odds ratios as approximations for adjusted relative risks in this calculation.

\section{Results \\ Descriptives}

Table 1 describes key outcomes and predictors for: (1) all singleton term births and (2) the sub-group of deliveries where outside uterotonic use was reported.

\section{Prevalence of outside uterotonic use}

$5.6 \%$ of the women reported using an outside uterotonic, either as an injection or drip prior to arriving at the CEmONC facility. Prolonged labor greatly increased the reported use of uterotonics. For labors reported as lasting less than $12 \mathrm{~h}, 5 \cdot 1 \%$ of women reported using outside uterotonics $(3.4 \%$ injections and $4.4 \%$ drip). For prolonged labors reported as lasting at least $12 \mathrm{~h}$, these proportions rose substantially: $14.7 \%$ reporting an outside uterotonic, $11.6 \%$ reporting injections specifically, $13 \cdot 0 \%$ reporting a drip specifically.

Association of outside uterotonic use with birth outcomes The crude odds ratios for the association of uterotonic use outside CEmONC with 3 of the 4 birth outcomes 
Table 1 Description of singleton term births

\begin{tabular}{lll}
\hline & $\begin{array}{l}\text { All Singleton term births } \geq 37 \text { weeks } \\
n=22426\end{array}$ & $\begin{array}{l}\text { Group reporting outside uterotonics } \\
n=1260\end{array}$ \\
\hline Perinatal Deaths & $361(1 \cdot 6 \%)$ & $63(5 \cdot 0 \%)$ \\
Fresh Still Births & $191(0 \cdot 9 \%)$ & $8(0 \cdot 6 \%)$ \\
Macerated Still Births & $5(0 \cdot 0 \%)$ & $3(0 \cdot 2 \%)$ \\
Unspecified Still Births & $318(1 \cdot 4 \%)$ & $53(4 \cdot 2 \%)$ \\
Total early Neonatal deaths & $229(1 \cdot 0 \%)$ & $41(3 \cdot 3 \%)$ \\
Neonatal deaths from Birth Asphyxia & $875(3 \cdot 9 \%)$ & $124(9 \cdot 8 \%)$ \\
Total Perinatal deaths & $1289(5 \cdot 7 \%)$ & $185(14 \cdot 4 \%)$ \\
Birth Complications & $63(0 \cdot 3 \%)$ & $5(0 \cdot 4 \%)$ \\
Prolonged labor (>12 h) & $303(1 \cdot 4 \%)$ & $12(1 \cdot 0 \%)$ \\
Pre-eclampsia (Moderate) & $261(1 \cdot 2 \%)$ & $13(1 \cdot 0 \%)$ \\
Pre-eclampsia (Severe) & & $484(38 \cdot 4 \%)$ \\
Eclampsia & $4953(21 \cdot 1 \%)$ & $97(7 \cdot 7 \%)$ \\
Hospital Procedures & $1501(6 \cdot 7 \%)$ & $435(34 \cdot 5 \%)$ \\
Cesarian-sections & $7516(33 \cdot 5 \%)$ & \\
Cesarians due to fetal compromise & & \\
Uterotonic to augment or induce &
\end{tabular}

$\%$ of births in parentheses

were substantial and statistically significant-Fresh stillbirth $\mathrm{OR}=3 \cdot 895 \% \mathrm{CI}(2 \cdot 8,4 \cdot 9)$, early NND OR $=2 \cdot 9$ $(2 \cdot 1,3 \cdot 9)$, early NND due to Birth Asphyxia OR $=3 \cdot 8$ $(2 \cdot 7,5 \cdot 3)$ (all $p<0.001)$. These are the birth outcomes that specifically relate to intrapartum events. The odds ratio for the remaining birth outcome, macerated stillbirth, which relates to the baby dying before the onset of labor was not found to be statistically associated with outside uterotonic use $\mathrm{OR}=0.7(0 \cdot 4,1 \cdot 5) \quad(p>0.10)$. The crude odds ratio for the composite birth outcome perinatal death was substantial and statistically significant $\mathrm{OR}=3 \cdot 0$ $(2 \cdot 4,3 \cdot 6)(p<0.001)$ (Table 2).

Table 3 presents the model-adjusted odds ratios. After controlling for all covariates, there is an increased risk of all categories of perinatal mortality if the mother had a diagnosis of either prolonged labor, moderate or severe pre-eclampsia, and eclampsia. There was no significant associated between LAMB Hospital administered uterotonics and any perinatal outcomes. There was a decreased

Table 2 Crude odds ratios for four perinatal outcomes and total perinatal mortality

\begin{tabular}{ll}
\hline & Odds Ratio $(95 \% \mathrm{Cl})$ \\
\hline Fresh Stillbirth & $3 \cdot 7^{*}(2 \cdot 8,4 \cdot 9)$ \\
Macerated Stillbirth & $0 \cdot 7(0 \cdot 4,1 \cdot 5)$ \\
Early NND & $2 \cdot 9^{*}(2 \cdot 1,3 \cdot 9)$ \\
Early NND due to Birth Asphyxia & $3 \cdot 8^{*}(2 \cdot 7,5 \cdot 3)$ \\
All Perinatal Death (Composite) & $3 \cdot 0^{*}(2 \cdot 4,3 \cdot 6)$
\end{tabular}

Notes: ${ }^{*} p<0.001$ risk of fresh stillbirth and an increased risk of early neonatal death and death due to birth asphyxia with Cesarian sections. Overall, Cesarian sections were associated with lower risk of perinatal mortality $(\mathrm{OR}=0.7(0.5,0.8)(p<0.001)$.

When adjusting for prolonged labor, pre-eclampsia, eclampsia, hospital administration of uterotonics, and Cesarian section, the associations of all perinatal mortality outcomes with outside uterotonic use remained significant and substantial, with the exception of macerated stillbirths (Table 3).

\section{Estimating population attributable risk}

If these statistical associations reflect the causal impact of outside uterotonic use on perinatal mortality with no unmeasured confounding or omitted variables, then the estimated percentage of deaths due to exposure to uterotonics outside the CEmONC setting is $10 \%$ of early neonatal deaths, $11 \%$ of birth asphyxia deaths, $10 \%$ of perinatal deaths and $14 \%$ of fresh stillbirths. These estimates are based on the current population with a relatively low rate of reported outside uterotonic use $(5.6 \%)$ compared to other published studies. If we adjust these estimates for higher rates of outside uterotonic use in other studies (10-68 \% of home births) [9-13], then such uterotonic use would be associated with much greater percentage of all perinatal mortality-for example $28 \%$ of perinatal deaths at $20 \%$ births with outside uterotonic use or $36 \%$ of perinatal deaths at $30 \%$ outside uterotonic use. 
Table 3 Logistic Regressions predicting five birth outcomes from reported outside uterotonics, adjusted odds ratios (singleton, term births $\geq 37$ weeks, $n=22426$ )

\begin{tabular}{|c|c|c|c|c|c|}
\hline \multirow[b]{2}{*}{ Predictor } & \multicolumn{5}{|l|}{ Outcome } \\
\hline & Fresh Stillbirth & Macerated Stillbirth & Early Neonatal Death & Intrapartum causes (Birth Asphyxia) & All Perinatal Deaths \\
\hline Outside uterotonics & $4 \cdot 0^{* * *}(3 \cdot 0,5 \cdot 3)$ & $0 \cdot 8(0 \cdot 4,1 \cdot 7)$ & $2 \cdot 9^{* * *}(2 \cdot 1,4 \cdot 0)$ & $3 \cdot 1^{* *}(2 \cdot 2,4 \cdot 5)$ & $3 \cdot 0^{* * *}(2 \cdot 4,3 \cdot 7)$ \\
\hline \multicolumn{6}{|l|}{ Labor duration } \\
\hline$<12 \mathrm{~h}$ & ref. & ref. & ref. & ref. & ref. \\
\hline $12-24 \mathrm{~h}$ & $3 \cdot 1^{* * *}(2 \cdot 1,4 \cdot 4)$ & $1 \cdot 9^{*}(1 \cdot 0,3 \cdot 5)$ & $1 \cdot 9^{* *}(1 \cdot 3,2 \cdot 9)$ & $2 \cdot 1^{* *}(1 \cdot 3,3 \cdot 3)$ & $2 \cdot 5^{* * *}(1.9,3.2)$ \\
\hline$>24 \mathrm{~h}$ & $2 \cdot 5^{* * *}(1 \cdot 5,4 \cdot 3)$ & $1 \cdot 7(0 \cdot 7,3 \cdot 8)$ & $1 \cdot 0(0 \cdot 4,2 \cdot 3)$ & $1 \cdot 1(0 \cdot 5,2 \cdot 8)$ & $1 \cdot 8^{* *}(1 \cdot 2,2 \cdot 6)$ \\
\hline Missing & $2 \cdot 4^{* * *}(1 \cdot 5,3 \cdot 9)$ & $2 \cdot 6^{*}(1 \cdot 1,6 \cdot 0)$ & $1 \cdot 3(0 \cdot 9,1 \cdot 9)$ & $1 \cdot 2(0 \cdot 8,1 \cdot 9)$ & $1 \cdot 7^{* * *}(1 \cdot 3,2 \cdot 3)$ \\
\hline \multicolumn{6}{|l|}{ Pre-eclampsia } \\
\hline none & ref. & ref. & ref. & ref. & ref. \\
\hline moderate & $2 \cdot 0(0.5,8.4)$ & $1 \cdot 7(0.2,12.2)$ & $2 \cdot 0(0.5,8.2)$ & $2 \cdot 8(0.7,11.5)$ & $2 \cdot 0(0.8,5.0)$ \\
\hline severe & $4 \cdot 5^{* * *}(2.7,7.6)$ & $3 \cdot 0^{* *}(1.5,6.3)$ & $2 \cdot 6^{* *}(1.4,4.7)$ & $3 \cdot 3^{* * *}(1.8,6.2)$ & $3 \cdot 6^{* * *}(2.5,5.1)$ \\
\hline Eclampsia & $4 \cdot 7^{* * *}(2.8,7.9)$ & NA & $4 \cdot 7^{* * *}(2.8,7.8)$ & $5 \cdot 6^{* * *}(3.2,9.7)$ & $3 \cdot 7^{* *}(2.5,5.4)$ \\
\hline \multicolumn{6}{|c|}{ Hospital-based Uterotonic Augmentation } \\
\hline No & ref. & ref. & ref. & ref. & ref. \\
\hline Yes & $1 \cdot 0(0 \cdot 5,1 \cdot 9)$ & $2 \cdot 4(0 \cdot 5,10 \cdot 9)$ & $0 \cdot 6(0 \cdot 4,1 \cdot 1)$ & $0 \cdot 7(0 \cdot 3,1 \cdot 4)$ & $0 \cdot 8(0 \cdot 5,1 \cdot 3)$ \\
\hline Unknown & $1 \cdot 2(0 \cdot 6,2 \cdot 5)$ & $5 \cdot 8^{*}(1 \cdot 3,27 \cdot 0)$ & $0 \cdot 8(0 \cdot 5,1 \cdot 5)$ & $1 \cdot 1(0 \cdot 5,2 \cdot 3)$ & $1 \cdot 3(0 \cdot 8,2 \cdot 0)$ \\
\hline \multicolumn{6}{|l|}{ Cesarian Section } \\
\hline No & ref. & ref. & ref. & ref. & ref. \\
\hline Yes & $0 \cdot 3^{* * *}(0 \cdot 2,0 \cdot 4)$ & $0 \cdot 1^{* * *}(0 \cdot 0,0 \cdot 2)$ & $2 \cdot 0^{* * *}(1 \cdot 6,2 \cdot 6)$ & $2 \cdot 1^{* * *}(1 \cdot 6,2 \cdot 8)$ & $0 \cdot 7^{* * *}(0 \cdot 5,0 \cdot 8)$ \\
\hline
\end{tabular}

Adjusted Odds Ratio with $95 \% \mathrm{Cl}$ in parentheses, adjusted for length of labor, hospital-based uterotonic augmentation or induction, pre-eclampsia, eclampsia, and Cesarian section

Note: ref reference category

${ }^{* * *} p<0.001,{ }^{* *} p<0.01,{ }^{*} p<0.05$

\section{Discussion}

In a CEmONC setting in Northwestern Bangladesh, we find that reported uterotonic use prior to arrival is associated with a substantially increased risk of perinatal mortality, including fresh stillbirth, early neonatal death prior to discharge, and death from birth asphyxia. This effect is independent of the potential confounding effect of prolonged labor, pre-eclampsia, eclampsia, CEmONCadministration of uterotonics and Cesarian section. Consistent with the expectation that outside uterotonic use increases the risk for perinatal mortality, there is no association with macerated stillbirth which would in most cases result from antepartum fetal death that precedes uterotonic use in labour. The findings about neonatal outcomes confirm results of a recent study in another South Asian setting showing similar relationships between unmonitored uterotonic use and neonatal mortality and morbidity [11]. However, by estimating the increased risk of fresh stillbirth associated with outside uterotonic use, our findings also highlight the potential impact of uterotonic use outside of CEmONC on all babies' lives during the entire continuum of intrapartum and postnatal life.

In this setting, we estimate that outside uterotonic use accounts for $10 \%$ of all perinatal deaths, including $11 \%$ of birth asphyxia deaths and $14 \%$ of fresh still births.
However, in both hospital- and community-based surveys conducted in the hospital catchment area, this population has substantially lower rates of reported outside uterotonic use than other populations in South Asia and low-income countries $(5.6 \%$ and 2.3 to $23.5 \%$ in representative surveys of recent births in the hospital source communities compared to $10-68 \%$ in other studies) [9-13]. If we assume outside uterotonic use is associated with the same risk of perinatal mortality as in a setting with higher rates of home-based births use uterotonics, then we estimate much higher proportions of perinatal deaths would be due to uterotonics outside a facility with appropriate controls in place (e.g., $28 \%$ of perinatal deaths with $20 \%$ use).

Although this study showed no evidence of increased risk from uterotonics administered after arrival at this CEmONC facility, it is important to point out that delivery in a facility designated as CEmONC may not be synonomous with appropriate control. Specifically, it is crucial to have controls in place for appropriate monitoring and regulation of the uterotonic infusion including, monitoring uterine contractions, fetal heartrate, use of the partograph as a decision making tool and immediate access to Cesarian section when needed. Not all CEmONC facilities may adhere to these standards in 
practice. These findings also show that even in a CEmONC facility with the capacity to provide a Cesarian-section within $20 \mathrm{~min}$ of admission, as is the case in this study, there are still a remarkable number of perinatal deaths which may be due to the sequelae of prior outside uterotonic use.

These findings must be interpreted in light of a number of data limitations. Reporting in locally meaningful language of "injections and drips to increase labor pains" is only a proxy for actual use of uterotonics outside the CEmONC facility. It is likely subject to underreporting to hospital staff due to either self-presentation bias on the part of patients or failure to ask and / or document on the part of staff. It is possible that some of the drips administered outside were plain intravenous fluids and did not contain uterotonics. Nonetheless, the proxy measure for uterotonic use is strongly associated in the expected direction with perinatal outcomes, suggesting that it is a suitably valid measure of outside uterotonic use. Assessing the stage in labour that the mother presented to the CEmONC hospital and at which stage the outside jnjectable uterotonics were used as well as the dose or frequency used were beyond the scope of this study.

The deliveries at LAMB hospital also represent a selective sample of all those deliveries in the surrounding area $[20,21]$. They likely include more complications and fewer normal deliveries than occur in the surrounding population, and thus leave open questions about the generalizability of the specific estimates or qualitative findings to the broader population. Recent efforts to monitor outside uterotonic use and perinatal mortality for all deliveries in the hospital's surrounding area will hopefully shed new light on the generalizability of these findings.

It is also possible that prolonged labor still confounds the relationship between outside uterotonic administration and perinatal mortality, but that error in assessing prolonged labor leads to incomplete detection of statistical confounding. However, the fact that adding an assessment of prolonged labor does almost nothing to change the estimated effect of outside uterotonic use on perinatal mortality partially alleviates this concern.

\section{Conclusions}

We find that reported use of uterotonics prior to arriving at the comprehensive facility is associated with a substantially increased risk of perinatal mortality. These findings point to the potentially substantial improvement in perinatal mortality to be made by reducing the currently widespread use of uterotonics for induction and augmentation outside of CEmONC facilities in low- and middle-income countries. Such efforts would benefit from systematic surveillance of such use as well as studies that identify and target the specific pathways by which uterotonics come to be administered outside of
CEmONC facilities. These will crucially involve a better understanding of the way that local availability, perceived medical need, and family demands for a quick delivery play a role in guiding decisions to use uterotonics outside of CEmONC facilities.

\section{Additional file}

Additional file 1: Perinatal mortality associated with use of uterotonics outside of Comprehensive Emergency Obstetric and Neonatal Care: a cross-sectional study. (DOCX $34 \mathrm{~kb}$ )

\section{Abbreviations \\ CEmONC: Comprehensive Emergency Obstetric Neonatal Care; early NND: early Neonatal Death (day 0-7); EmONC: Emergency Obstetric and Neonatal Care; FISH: Flow Information System Hospital database; MIS-Research: Management Information Systems - Research Department; PPIP: Perinatal Problem Identification Programme}

\section{Acknowledgements \\ None.}

\section{Funding}

No funding was received for this work.

Availability of data and materials

Data is available by discussion with the authors.

Authors' contributions

Both DH and LTD contributed equally to the paper: study conception design, data analysis, literature review and manuscript preparation, FM and SLS contributed to study conception. SLS and EJ contributed to literature review. LTD and SA designed database and with EJ data cleaning. DH ran the statistical analysis. All authors read and approved the final version before submission.

Competing interests

The authors declare that they have any competing interests.

Consent for publication

Not applicable.

\section{Ethics approval and consent to participate}

The study was approved as a retrospective analysis of anonymized hospital records by LAMB Research Ethics Committee (Parbatipur, Bangladesh). Given the retrospective nature of the study, it was not possible to seek consent from the individual patients.

\section{Author details}

${ }^{1}$ LAMB MIS-Research Department, Parbatipur, Dinajpur 5250, Bangladesh. ${ }^{2}$ LAMB Hospital Pediatric Department, Parbatipur, Dinajpur 5250, Bangladesh. ${ }^{3}$ Arizona State University, Tempe, AZ 85287, USA. ${ }^{4}$ LAMB Hospital Obstetric Department, Parbatipur, Dinajpur 5250, Bangladesh.

Received: 6 April 2016 Accepted: 16 September 2016 Published online: 06 October 2016

\section{References}

1. UNICEF. Child_Mortality_Report_2015_Web_8_Sept_15.pdf http://www.unicef. org/publications/files/Child_Mortality_Report_2015_Web_8_Sept_15.pdf. Accessed 8 Sept 2015.

2. Blencowe H, Cousens S, Bianchi Jassir F, Say L, Chou D, Colin Mathers C, et al. National, regional, and worldwide estimates of stillbirth rates in 2015 with trends from 2000: a systematic analysis. Lancet Glob Health. 2016;4: e98-108. Published Online January 18, 2016. http://dx.doi.org/10.1016/ S2214-109X(15)00275-2. 
3. Lawn JE, Blencowe H, Waisa P, Amouzou A, Mathers C, Hogan D, et al. Stillbirths: rates, risk factors and acceleration towards 2013. Published Online January 18, 2016 http://dx.doi.org/10.1016/ S0140-6736(15)00837-5.

4. Oestergaard MZ, Inoue M, Yoshida S, Mahanani WR, Gore FM, Cousens S, et al. Neonatal mortality levels for 193 countries in 2009 with trends since 1990: a systematic analysis of progress, projections, and priorities. PLoS Med. 2011;8(8):e1001080.

5. Liu L, Oza S, Hogan D, Perin J, Rudan I, Lawn JE, et al. Global, regional, and national causes of child mortality in 2000-13, with projections to inform post-2015 priorities: an updated systematic analysis. The Lancet. 2014. Epub Sept. 30, 2014

6. Lawn JE, Blencowe H, Pattinson R, Cousens S, Kumar R, Ibiebele I, et al. Stillbirths: Where? When? Why? How to make the data count? Lancet. 2011;377(9775):1448-63.

7. Darmstadt GL, Kinney MV, Chopra M, Cousens S, Kak L, Paul VK, et al. Who has been caring for the baby? Lancet. 2014;384:174-88. Published Online May 20, 2014 http://dx.doi.org/10.1016/S0140-6736(14)60458-X.

8. de Bernis L, Vinney MV, Stones W, ten Hoope-Bender P, Vivio D, Susannah Hopkins Leisher S, et al. Stillbirths: ending preventable deaths by 2030. The Lancet. Published Online January 18, 2016 http://dx.doi.org/10.1016/ S01406736(15)00954-X.

9. Lovold A, Stanton C, Armbruster D. How to avoid iatrogenic morbidity and mortality while increasing availability of oxytocin and misoprostol for PPH prevention? Int J of Gynecol Obstet. 2008;103(3):276-82.

10. Jeffery P, Das A, Dasgupta J, Jeffery R. Unmonitored intrapartum oxytocin use in home deliveries: evidence from Uttar Pradesh, India. Reprod Health Matters. 2007;15(30):172-8.

11. Mullany LC, Khatry SK, Katz J, Stanton CK, Lee AC, Darmstadt GL, et al. Injections during labor and intrapartum-related hypoxic injury and mortality in rural southern Nepal. Int J Gynecol Obstet. 2013;122(1):22-6.

12. Moran A, Wahed T, Afsana K. Oxytocin to augment labor during home births: an exploratory study in the urban slums of Dhaka, Bangladesh. BJOG. 2010;117(13):1608-15.

13. lyengar SD, lyengar K, Suhalka V, Agarwal K. Comparison of domiciliary and institutional delivery-care practices in rural Rajasthan, India. J Health Popul Nutr. 2009;27(2):303.

14. Brhlikova P, Jeffery P, Bhatia GP, Khurana S. Intrapartum oxytocin (mis) use in South Asia. J Health Stud. 2009;2:33-50.

15. Flandermeyer D, Stanton C, Armbruster D. Uterotonic use at home births in low-income countries: a literature review. Int J Gynecol Obstet. 2010;108(3):269-75.

16. Finnström O. Studies on maturity in newborn infants IX. Further observations on the use of external characteristics in estimating gestational age. Acta Paediatr. 1977;66(5):601-4.

17. PPIP. Perinatal Problem Identification Program (PPIP v3). www.ppip.co.za 2013 [29 Apr 2013].

18. Day LT, Alam S, Mondal N, Mollick B. Implementation of an integrated hospital information system in limited-resource setting in rural Bangladesh. ASCON, Annual Scientific Conference, International Centre Diarrhoeal Disease Research; Dhaka, Bangladesh; 2011.

19. Rockhill B, Newman B, Weinberg C. Use and misuse of population attributable fractions. Am J Public Health. 1998;88(1):15-9.

20. Jeffery P, Jeffery R. 'Money itself discriminates' Obstetric emergencies in the time of liberalisation. Contrib Indian Soc. 2008:42(1):59-91.

21. Jeffery P, Jeffery R. Only when the boat has started sinking: A maternal death in rural north India. Soc Sci Med. 2010;71(10):1711-8.

\section{Submit your next manuscript to BioMed Central and we will help you at every step:}

- We accept pre-submission inquiries

- Our selector tool helps you to find the most relevant journal

- We provide round the clock customer support

- Convenient online submission

- Thorough peer review

- Inclusion in PubMed and all major indexing services

- Maximum visibility for your research

Submit your manuscript at www.biomedcentral.com/submit
Biomed Central 